\title{
Design nonlinear model reference with fuzzy controller for nonlinear SISO second order systems
}

\author{
Ekhlas H. Karam ${ }^{1}$, Nasir A. Al-Awad ${ }^{2}$, Noor S. Abdul-Jaleel ${ }^{3}$ \\ ${ }_{1,2}$ Department of Computer Engineering, Al-Mustansiriyah University, Iraq \\ ${ }^{3}$ Department of Electrical Engineering, Al-Mustansiriyah University, Iraq
}

\begin{tabular}{l} 
Article Info \\
\hline Article history: \\
Received Jun 16, 2018 \\
Revised Jan 8, 2019 \\
Accepted Jan 11, 2019 \\
\hline Keywords: \\
Model reference controller \\
Inverted pendulum type-2 \\
Fuzzy controller \\
Takagi-Sugeno type
\end{tabular}

Article Info

Article history

Received Jun 16, 2018

Revised Jan 8, 2019

Accepted Jan 11, 2019

\section{ords:}

Inverted pendulum type-2

Takagi-Sugeno type

\begin{abstract}
Model reference controller is considering as one of the most useful controller to specific performance of systems where the desired output is produced for a given input. This system used the difference between the outputs of the plant and the desired model by comparing them to produce the signals of the control. This paper focus on design a model reference controller (MRC) combined with (type-1 and interval type-2) fuzzy control scheme for single input-single output (SISO) systems under uncertainty and external disturbance. The model reference controller is designed firstly without fuzzy scheme based on an optimal desired model and Lyapunov stability theory. Then a (type-1 and Interval type-2) fuzzy controller Takagi-Sugeno type is combine with the suggested MRC in order to enhance the performer of it, the common parts between the two fuzzy systems such as: fuzzifier, inference engine, fuzzy rule-base and defuzzifier are illustrated. In this paper the proposed controller is applied to controla (SISO) inverted pendulum sustem and the Matlab R2015 software is used to carry out two simulation cases for the overall controlled scheme. The obtained results for the two cases show that the proposed MRC with both fuzzy control schemes have acceptable performance, but it have better performance with the interval type-2 fuzzy scheme.
\end{abstract}

Copyright $@ 2019$ Institute of Advanced Engineering and Science. All rights reserved.

\section{Corresponding Author:}

Noor S. Abdul-Jaleel,

Department of Electrical Engineering, Faculty of Engineering,

Al-Mustansiriyah University, Iraq.

Email: mail22ns@uomustansiriyah.edu.iq

\section{INTRODUCTION}

Control of nonlinear process is main criteria in the process control industries. This kind of nonlinear process gives many control problems (uncertain and time varying parameters) due to their nonlinearities [1,2]. For nonlinear systems design, numerous methods were introduced. These can be subdivided into techniques which attempt to treat the system as a linear system in a limited range of operation and use (well-known) linear design techniques for each region, or those that attempt to introduce auxiliary nonlinear feedback in such a way that the system treated as linear for purposes of control design, such as Feedback linearization, Lyapunov based methods, Lyapunov Redesign, Back-stepping, sliding mode control [3]. Nonlinear controllers can be proposed using methods very well-known from the theory of linear systems [4].

It is known that, classical methods for design controller to nonlinear systems, needs re-tune the controller and this tuning is complicated, so an adaptive procedures are necessary [5]. Furthermore, control of nonlinear systems by feedback linearization is well known and has been applied to control of a wide variety of nonlinear dynamic systems, but needs more tuning [6].

Fuzzy logic controllers (FLC's) are one of useful control schemes for nonlinear systems or systems having difficulties in deriving mathematical models or having performance limitations with conventional linear control schemes [7]. Most works in fuzzy control field for use the Type-1 fuzzy logic systems (FLSs) 
such in [8-10], these systems are known for their ability to compensate for structured and unstructured uncertainties, to a certain degree. However, type-2 fuzzy engines have been credited to be more powerful in compensating for even higher degrees of uncertainties [11]. They are particularly suitable for time-variant systems with unknown time- varying dynamics. They also allow for more flexibility to alleviate the problems associated to the uncertainties pertaining to the choice of the system's fuzzy rules and fuzzy membership functions [12]. Recently, many researches have been done on system control and stability analysis based on type-2 fuzzy systems such in [13-15] because these researchers shown that type-1 fuzzy logic control have difficulty in modeling and minimizing the effect of uncertainties [16, 17].

In other word, one of adaptive and robust schemes that are used to control the nonlinear system is the model reference control (MRC) which popular in the area of self-tuning control, In the MRC, the error between the reference model output and the real plant output is used to adjust its parameters in order to control the plant to follow the desired output from the reference model [18].

The performance of MRC can be improved by additional controllers like in [19] which suggest a fuzzy model reference learning control (FMRLC) to improve the existing learning control approaches or introducing new ones because the fuzzy controller constructed for the nominal plant may perform inadequately if significant and unpredictable plant parameter variations. While in [20], the feedback linearization, the conventional model reference control, interval type-2 fuzzy scheme (IT2FLS) and the idea of the sliding mode control method are combined to propose a hybrid control system to address the problem of the conventional model reference control and resolve the uncertain hyperchaotic system tracking problem.

In this paper, a model reference controller combined with adaptive (type-1 and interval type-2) fuzzy control scheme, is designed for stabilization of SISO nonlinear second order systems, working in the presence of uncertainties and external disturbances. The updates for the MRC are performed using adaptation laws derived from the stability study in the Lyapunov sense. The adaptive (type-1, interval type-2) fuzzy control scheme is used to improve the performance of the MRC by reduce error steady state from pendulum response. The organization of this paper is as follows. In section 2, the problem formulation and the details for the adaptive model reference controller design are explained. A briefly introduction for type-1 and interval type-2 fuzzy is given in section 3. The combination of MRC with fuzzy control scheme design is addressed in section 4 . Section 5 demonstrate the efficiently of the proposed controller by simulation results. Finally, the summary and conclusions are given in section 6.

\section{PROBLEM FORMULATION AND MODEL-REFERENCE CONTROLLER DESIGN}

The general class of single-input and single-output (SISO) uncertain dynamic nonlinear systems can be described by the following mathematical equation [21, 22]:

$$
\begin{aligned}
& x^{(n)}=f(x)+b(x) u(t)+d(t) \\
& y=x
\end{aligned}
$$

where $x=\left(x, \dot{x}, \cdots, x^{(n-1)}\right)^{T} \in R$ is the state vector of the system, $f(x)$ is unknown continuous nonlinear uncertain function, $\mathrm{g}(x)$ is the control gain function, $u(t) \in R$ is the input of the system and $y(t) \in R$ is system output, unknown nonlinear uncertain function of the system, and $d(t)$ is the external unknown disturbance.

The problem of controller design is state as follows. If the desired trajectories $x_{d}, \dot{x}_{d}, \ddot{x}_{d}\left(x_{d} \in R^{n}\right.$ is bounded) are given with all (or some) system states being unknown, then derive a low of controller for the force (or torque) input to make the states of the system to flow a desired states $x_{d}=\left(x_{d}, \dot{x}_{d}, \cdots, x^{(n-1)}\right)^{T}$ in the presence of external disturbances and model uncertainties, closely if not very exactly.

A robust and adaptive control law is needed to deal with system uncertainties, external disturbances and the rounding errors. To achive this objective, we suggest to use model reference controller (MRC) for nonlinear second order systems $(n=2)$. The model-reference controller can be considered as one of the most useful methods to specific performance system where the desired output is produced for a given input. This controller can be simulated as mathematical model on a computer without needing of practical hardware and the controller used the difference between the outputs of the plant and the model by comparing them to produce the signals of the control [23]. The model of the nonlinear system in (1) with $(n=2)$ can be written as the state equation:

$$
\dot{x}=f_{p}(x, u, t)
$$


or can be written as:

$$
\left[\begin{array}{l}
\dot{x}_{1} \\
\dot{x}_{2}
\end{array}\right]=\left[\begin{array}{ll}
0 & 1 \\
0 & 0
\end{array}\right]\left[\begin{array}{l}
x_{1} \\
x_{2}
\end{array}\right]+\left[\begin{array}{l}
0 \\
1
\end{array}\right](f+g u+d), y=\left[\begin{array}{ll}
1 & 0
\end{array}\right]\left[\begin{array}{l}
x_{1} \\
x_{2}
\end{array}\right]
$$

where $x=$ state vector ( 2 -state), $u$ is single control signal, $f$ is nonlinear function, $d$ is the system perturbation. The procedure for designing MRC is given by the following steps:

Step 1: define the desired model equation by:

$$
\dot{x}_{d}=A x_{d}+B v
$$

where $x_{d}$ is the state vector of the desired model, $v$ is the desired input signal, $A$ is $2 \times 2$ constant matrix, and $B$ is $2 \times 1$ constant matrix. The system of model-reference has an asymptotically stable equilibrium state, when the eigenvalues of $(A)$ have negative real parts. In this paper the equation of the desired reference model chosen as:

$$
\left[\begin{array}{l}
\dot{x}_{d 1} \\
\dot{x}_{d 2}
\end{array}\right]=\left[\begin{array}{cc}
0 & 1 \\
-\omega_{n}^{2} & -2 \zeta \omega_{n}
\end{array}\right]\left[\begin{array}{l}
x_{d 1} \\
x_{d 2}
\end{array}\right]+\left[\begin{array}{c}
0 \\
\omega_{n}^{2}
\end{array}\right] v
$$

where $\omega_{n}$ is desired natural frequency, and $\zeta$ is damping ratio.

Step 2: define the error vector $(e)$ by:

$$
e=x_{d}-x
$$

If the error vector was reduced to zero with a suitable value of $(u)$ :

$$
\begin{aligned}
\dot{e} & =\dot{x}_{d}-\dot{x}=A x_{d}+B v-f_{p}(x, u, t) \\
& =A e+A x-f_{p}(x, u, t)+B v
\end{aligned}
$$

The above equation represents the differential equation for the error vector.

Step 3: assume that the function of Lyapunov can be written as:

$$
V(e)=e^{T} P e
$$

where $P=$ a positive-definite real, symmetric matrix. By taken the derivative of $V(e)$ with time respect:

$$
\begin{aligned}
\dot{V}(e) & =\dot{e}^{T} P e+e^{T} P \dot{e} \\
& =\left[e^{T} A^{T}+x^{T} A^{T}-f^{T}(x, u, t)+v^{T} B^{T}\right] P e+e^{T} p\left[A e+A x-f_{p}(x, u, t)+B v\right] \\
& =e^{T}\left(A^{T} P+P A\right) e+2 M
\end{aligned}
$$

where

$$
M=e^{T} P\left[A x-f_{p}(x, u, t)+B v\right]
$$

Since $A^{T} P+P A=-Q$, so Equation (9) can be written as:

$$
\dot{V}(e)=-e^{T} Q e+2 M
$$

The $V(e)$ is assumed Lyapunov function if $(-Q)$ is a negative definite matrix and the control signal $u(t)$ should be chosen such that the scalar $M$ is negative [20]. When $V(e) \rightarrow \infty$ as $\|e\| \rightarrow \infty$, then the equilibrium state $\left(e_{1}, e_{2}\right)=(0,0)$ is a symptically stable in large.

In order to satisfy Equation (11), The $Q$ matrix is chosen as diagonal matrix:

$$
Q=\left[\begin{array}{cc}
q_{11} & 0 \\
0 & q_{22}
\end{array}\right]=\text { positive definite }
$$

The rate of convergence of transient response depends on matrix $P$, which in turn depends on matrix $Q$, so $\dot{V}$ becomes;

$$
\dot{V}(e)=-\left(q_{11} e_{1}^{2}+q_{22} e_{2}^{2}\right)+2 M
$$


According to second order model (Equation (3)) and the desired model (Equation (5)), the scalar $M$ is:

$$
\begin{aligned}
M & =\left[\begin{array}{ll}
e_{1} & e_{2}
\end{array}\right]\left[\begin{array}{ll}
p_{11} & p_{12} \\
p_{21} & p_{22}
\end{array}\right]\left(\left[\begin{array}{cc}
0 & 1 \\
-\omega_{n}^{2} & -2 \zeta \omega_{n}
\end{array}\right]\left[\begin{array}{l}
x_{1} \\
x_{2}
\end{array}\right]-\left[\begin{array}{cc}
0 & 1 \\
0 & 0
\end{array}\right]-\left[\begin{array}{c}
0 \\
g u
\end{array}\right]+\left[\begin{array}{c}
0 \\
\omega_{n}^{2} v
\end{array}\right]-\left[\begin{array}{l}
0 \\
f
\end{array}\right]\right) \\
& =\left(e_{1} p_{12}+e_{2} p_{22}\right)\left[-\omega_{n}^{2} x_{1}-2 \zeta \omega_{n} x_{2}+\omega_{n}^{2} v-g u-f\right]
\end{aligned}
$$

Here, if we chose $u$ for our design as:

$$
u=\left[-\omega_{n}^{2} x_{1}-2 \zeta \omega_{n} x_{2}+\left(k \tanh \left(e_{i} p_{12}+e_{2} p_{22}\right)\right)+\omega_{n}^{2} v-f\right] / g
$$

where tanh (.) (hyberbolic tangent) and $k$ is suitable constant. With Equation (14) the scalar $M$ is becomes:

$$
\begin{aligned}
M & =\left(e_{i} p_{12}+e_{2} p_{22}\right)\left[f-k \tanh \left(e_{i} p_{12}+e_{2} p_{22}\right)\right] \\
& =\text { nonpositive }
\end{aligned}
$$

\section{TYPE-1 AND TYPE-2 FUZZY SYSTEMS}

Type-1 fuzzy logic systems (FLSs) are known as the universal approximates and widely used to solve the real problems because it is capable of compensate the structure and unstructured uncertainties, but only to a certain degree, because some of the designed approaches face high level of uncertainties which effect on systems performances and since type-1 fuzzy system use crisp type-1 fuzzy sets in representation of fuzzy rules, so its cannot process the high level of uncertainties which associated with knowledge bases data or information.

The system of type-2 fuzzy that suggested by Zadeh and developed from Mendel with his coauthors [24-26] could make additional proficient over compensating that higher level for uncertainties because the membership functions of this system are themselves fuzzy. This type of fuzzy considered as one of more suitable systems that has unknown time-varying dynamics [27]. Interval type-2 fuzzy systems (IT2FSs) is consider as one a special state of T2FSs. because of the reduction load of computational [28], IT2FSs are used actually at different engineering areas [29],[30].

The IT2FSs structure is consists of as shown in Figure 1 (fuzzifier, rule base, inference engine, type reducer, and defuzzifier). It is similarity of T1FLS, only the main difference between them was happened in the output processing which contains a reducer form and a defuzzifier to produce the output signal from the type reducer or a crisp value from the defuzzifier [28]. So, the reduction type can take information on the rule of uncertainties more than the value of defuzzified. There are two main structures for an IT2 FLC as for a (T1FLC), Mamdani and Takagi-Sugeno-Kang (TSK). TSK is covered in this Section.

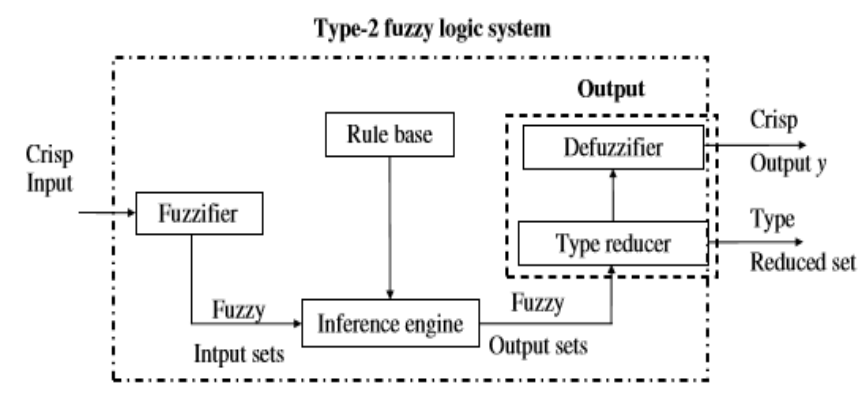

Figure 1. Type 2 fuzzy system [31]

The outputs definition with the consequent part of rules is the main difference between the type-1 TSK fuzzy logic controller [32] and a Mamdani type-1 fuzzy logic controller. If we consider the first order type-1 TSK fuzzy logic has $p$ inputs $x_{l} \in X_{1}, \ldots, x p \in X p$ and y one output $\epsilon Y$, and are the $M$ rules. The rule of $i$ th can be expressed as:

$$
\begin{aligned}
& R^{l}=I F x_{l} \text { is } \hat{F}_{1}^{l} \text { and } \ldots \text { and } x_{p} \text { is } \hat{F}_{p}^{l} \\
& \text { THEN } y^{l}=c_{0}^{l}+c_{0}^{l} x_{1}+\ldots+c_{p}^{l} x_{p}
\end{aligned}
$$


A type-2 TSK fuzzy logic controller or system (T2TSK fuzzy logic) was firstly suggested by Liang and Mendel [33]. In spite of the fact that T1TSK fuzzy systems gained a considerable measure attention, the searches around TSK type-2 fuzzy systems still little. T2TSK systems were applied by Liang and Mendel in channel equalization of channels [34]. Where, according to them, there are three models of T2TSK fuzzy logics which depending on the type of the antecedent and consequent part of rules, to have: T2TSK- Model I, T2TSK-Model II and T2TSK-Model III.

Tables 1 and 2 show the difference between T2TSK-Model II and T2TSK-Model III, where: $c_{i}^{l}, C_{i}^{l}=$ the consequent parameters,

$y_{i}^{l}, Y_{i}^{l}=$ the outputs of $i$ th rule

$\hat{F}_{j}^{l}(j=1 \ldots p)=$ type- 2 fuzzy sets

$\hat{F}_{j}^{l}(j=1 \ldots p)=$ type- 1 fuzzy sets

Table 1. Models of T2 Tsk FLS

\begin{tabular}{lll}
\hline Model & Antecedents & Consequents \\
\hline Model I & Type-2 fuzzy sets & Type-1 fuzzy sets \\
Model II & Type-2 fuzzy sets & Crisp numbers \\
Model III & Type-1 fuzzy sets & Type-1 fuzzy sets \\
\hline
\end{tabular}

Table 2. Rules of T2 TSK FLS

\begin{tabular}{ll}
\hline TSK FLS & Rules $R^{l}$ \\
\hline Type-1 & $I F x_{I}$ is $F_{1}^{l}$ and $\ldots$ and $x_{p} i s F_{p}^{l}$ \\
& THEN $y^{l}=c_{0}^{l}+c_{1}^{l} x_{1}+L+c_{p}^{l} x_{p}$ \\
T2 Model I & $I F x_{1}$ is $\tilde{F}_{1}^{l}$ and $\ldots$ and $x_{p} i s \tilde{F}_{p}^{l}$ \\
& THEN $Y^{l}=c_{0}^{l}+c_{1}^{l} x_{1}+\ldots+c_{p}^{l} x_{p}$ \\
T2 Model II & $I F x_{1}$ is $\tilde{F}_{1}^{l}$ and $\ldots$ and $x_{p} i s \tilde{F}_{p}^{l}$ \\
& THEN $y^{l}=c_{0}^{l}+c_{1}^{l} x_{1}+\ldots+c_{p}^{l} x_{p}$ \\
T2 Model III & $I F x_{I}$ is $F_{1}^{l}$ and $\ldots$ and $x_{p} i s F_{p}^{l}$ \\
& THEN $Y^{l}=c_{0}^{l}+c_{1}^{l} x_{1}+\ldots+c_{p}^{l} x_{p}$ \\
\hline
\end{tabular}

The firing strength of $i$ th rule $W^{i}(x)$ with the operation meet under product or lower $t$-norm is an interval type-1 set expressed as follows:

$$
\begin{aligned}
& W^{i}(x)=\left[\underline{W}^{i}(x), \bar{W}^{i}(x)\right] \\
& \underline{W}^{i}(x)=\underline{\mu} F_{1}^{i}\left(x_{1}\right) * \ldots * \mu F_{p}^{i}\left(x_{p}\right) \\
& \bar{W}^{i}(x)=\bar{\mu} F_{1}^{i}\left(x_{1}\right) * \ldots * \bar{\mu} F_{p}^{i}\left(x_{p}\right)
\end{aligned}
$$

Also the final output is a set of type-1 interval, and is calculated as follows:

For each rule we will acquire $y_{l}$ and $y_{r}$. Since all sets are crisp (18) results to:

$$
y_{1}=\frac{\sum_{i=1}^{M} w_{l}^{i} y_{l}^{i}}{\sum_{i=1}^{M} w_{l}^{i}}, y_{r}=\frac{\sum_{i=1}^{M} w_{l}^{i} y_{l}^{i}}{\sum_{i=1}^{M} w_{r}^{i}}
$$

where $y_{i} \in Y^{i}, Y^{i}=\left[y_{l}^{i}, y_{r}^{i}\right](i=1 \ldots M)$, the defuzzified output is:

$$
y=\left(y_{l}+y_{r}\right) / 2
$$




\section{MODEL REFERENCE CONTROLLER COMBINED WITH FUZZY SYSTEM}

The use of linear desired model in designing the model reference controller make the output of the inverted pendulum follow the desired input with good performance but there is still an error steady state. In order to enhance the efficiency of the model reference controller, a fuzzy control scheme is combined with MRC, where this scheme reduces the error steady state and the effect of model uncertainty and external disturbance. There are many efficient fuzzy controllers design with different $n * n$ rules.

In this paper we suggest to use simple PI TSK fuzzy controller with two inputs (error, integral of error) and one output $u_{f}$ as shown in Figure 2, where $K_{i}, K_{f}$ is integral and fuzzy output gains in respectively.

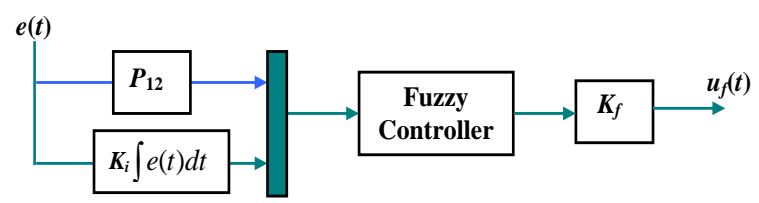

Figure 2. Fuzzy PI controller structure

The new control action $u_{n}$ is results from combine the fuzzy PI controller $u_{f}(t)$ with the suggested control action $u$ as:

$$
u_{n}=u+\left(\frac{u_{f}}{g}\right)
$$

or

$$
u_{n}=\left[-\omega_{n}^{2} x_{1}-2 \zeta \omega_{n} x_{2}+\left(k \tanh \left(e_{i} p_{12}+e_{2} p_{22}\right)\right)+u_{f}+\omega_{n}^{2} v-f\right] / g
$$

Hence the scalar $M$ is becomes:

$$
\begin{aligned}
M & =\left(e_{i} p_{12}+e_{2} p_{22}\right)\left[f-k \tanh \left(e_{i} p_{12}+e_{2} p_{22}\right)-u_{f}\right] \\
& =\text { nonpositive }
\end{aligned}
$$

We have followed the standard system of fuzzy controller's outline, which comprises of fuzzification, control rule base foundation, and defuzzification.

\subsection{Fuzzification}

The fuzzy PI controller has two input (error signal, integral of error) and one output $u_{f}$, the membership functions for the input and output variables of this scheme for type- 1 and type- 2 are ( $\mathrm{P}$ is positive, $\mathrm{Z}$ is zero, and $\mathrm{N}$ is negative) for the input of type- 1 as shown in Figure 3(a) and (NU is negative upper, $\mathrm{NL}$ is negative lower, $\mathrm{ZU}$ is zero upper, $\mathrm{ZL}$ is zero lower, $\mathrm{PU}$ is positive upper and PL is positive lower) for the input of type-2 as Figure 3(b) and five memberships (PB is positive big, PM is positive medium, $\mathrm{Z}$ is zero, NM is negative mediums, and NB is negative big) in output for both fuzzy types as shown in Figure 3(c).

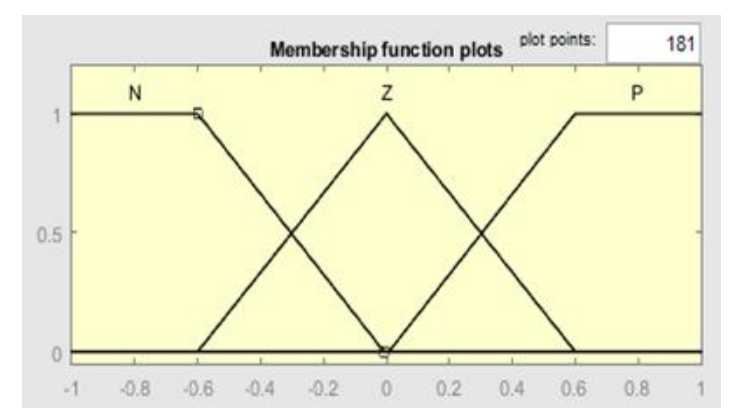

(a)

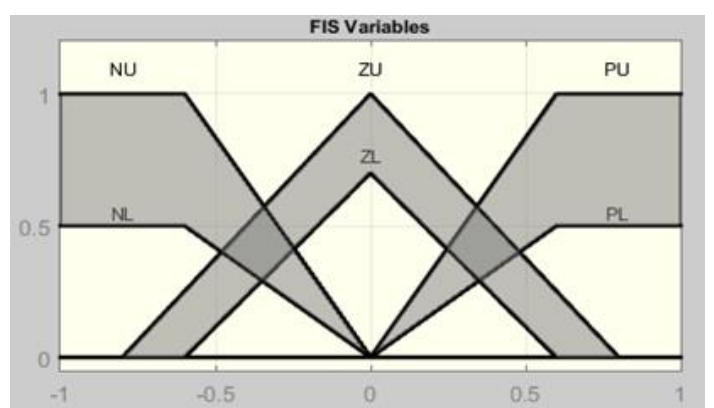

(b)

Figure 3. Membership function for, (a) input variable for type-1 fuzzy, (b) input variable for interval type-2 


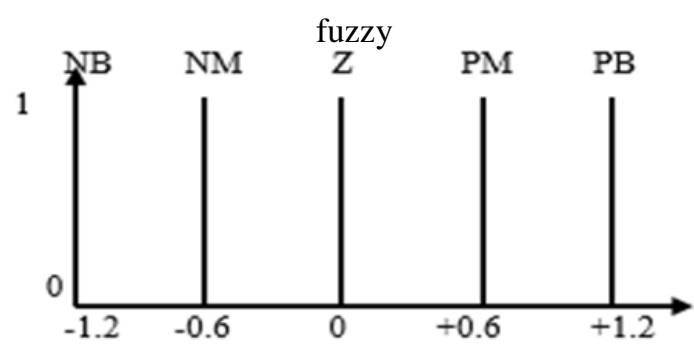

(c)

Figure 3. Membership function for, (c) output variable

\subsection{The rules of fuzzy control}

Utilizing the previously mentioned participation functions, the accompanying control rules are built up for the fuzzy PI controller.

$$
\left(R_{i}\right) \text { IF }\left(i / p_{1}\right) \text { is --- AND }\left(i / p_{2}\right) \text { is ---, THEN fuzzy PI output is ---. }
$$

where $i / p_{1}$ is the error signal, $i / p_{2}$ is integral of error signal. The logical "AND" takes the minimum. Table 3 [35]-[37], shows the rules tabular. If the number of output membership is reduce to three only then the above table can be modified to Table 4 .

Table 3. Rules of PI fuzzy controller

\begin{tabular}{cccc}
\hline$\Delta \mathrm{e}$ & \multicolumn{3}{c}{$\mathrm{e}$} \\
\cline { 2 - 4 } & $\mathrm{P}$ & $\mathrm{Z}$ & $\mathrm{N}$ \\
\hline $\mathrm{P}$ & $\mathrm{PB}$ & $\mathrm{PM}$ & $\mathrm{Z}$ \\
$\mathrm{Z}$ & $\mathrm{PM}$ & $\mathrm{Z}$ & $\mathrm{NM}$ \\
$\mathrm{N}$ & $\mathrm{Z}$ & $\mathrm{NM}$ & $\mathrm{NB}$ \\
\hline
\end{tabular}

Table 4. Reduction rules of PI fuzzy controller

\begin{tabular}{cccc}
\hline$\Delta \mathrm{e}$ & \multicolumn{3}{c}{$\mathrm{e}$} \\
\cline { 2 - 4 } & $\mathrm{N}$ & $\mathrm{Z}$ & $\mathrm{P}$ \\
\hline $\mathrm{N}$ & $\mathrm{N}$ & $\mathrm{N}$ & $\mathrm{Z}$ \\
$\mathrm{Z}$ & $\mathrm{N}$ & $\mathrm{N}$ & $\mathrm{P}$ \\
$\mathrm{P}$ & $\mathrm{Z}$ & $\mathrm{P}$ & $\mathrm{P}$ \\
\hline
\end{tabular}

\subsection{Reduction and defuzzfication}

To compute the output value, it's needed to calculate the main fuzzy type-2 by (18) and (19).

\section{SIMULATION RESULTS ANALYSIS AND COMPARISON}

In this section, the second order inverted pendulum system which is shown in Figure 4 is considered to show the efficiency of the proposed controller schem. Inverted pendulum is a traditional problem in dynamics systems and control theory, it is used mainly to test control algorithms. The main feature of this system is characterized by: unstable, nonlinear and the phase is non-minimum, which is lead to make the system more robustly and stability edge, made it the focus of attention of many researchers. So, most of the complex systems are used the inverted pendulum as a benchmark to control the system with different controller techniques [38].

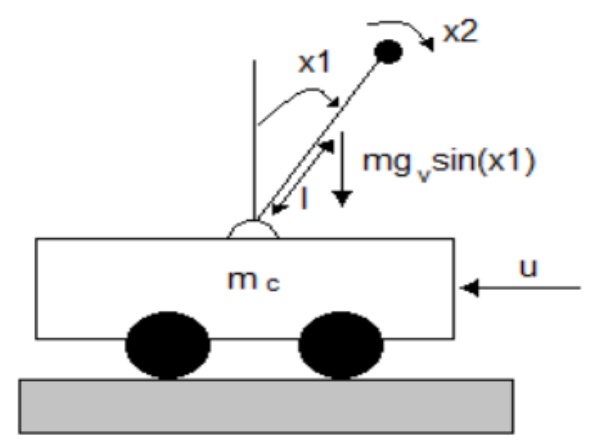

Figure 4. The System of the inverted 
The angle of the inverted pendulum was chosen as $\left(x_{1}=\theta\right)$ regarding to the vertical line. So, the equations for this inverted pendulum system can be written as [39-44]:

$$
\begin{aligned}
& \dot{x}_{1}=x_{2} \\
& \dot{x}_{2}=f+g u+d, y=x_{1}
\end{aligned}
$$

where

$$
f=\frac{g_{v} \sin x_{1}-\left(\frac{m l x_{2}^{2} \cos x_{1} \sin x_{1}}{m_{c}+m}\right)}{l\left(\frac{4}{3}-\frac{m \cos ^{2} x_{1 v}}{m_{c}+m}\right)}, \quad g=\frac{\frac{\cos x_{1}}{m_{c}+m}}{l\left(\frac{4}{3}-\frac{m \cos ^{2} x_{1}}{m_{c}+m}\right)} .>0
$$

In this paper, $d$ is define by [43]:

$$
d=7 \sin \left(10 x_{1}\right)+\cos x_{2}
$$

The parameters of inverted pendulum are given by Table 5 [43, 45].

Table 5. Parameters inverted pendulum

\begin{tabular}{clc}
\hline Symbol & \multicolumn{1}{c}{ Description } & Values \\
\hline$g_{v}$ & acceleration due to gravity & $9.8 \mathrm{~m} / \mathrm{sec}^{2}$ \\
$m_{c}$ & mass of the cart & $1 \mathrm{Kg}$ \\
$l$ & the half-length of the pole & $0.5 \mathrm{~m}$ \\
$u$ & control input & $\ldots \ldots \ldots$ \\
$m$ & the mass of the pole & $0.1 \mathrm{Kg}$ \\
\hline
\end{tabular}

By using the facility available in the Matlab software version (R2015), different simulations of the inverted pendulum model are carried out for linear and nonlinear trajectories are considered to illustrate the efficiency of the proposed control scheme. Figure 5 show the Matlab Simulink connection for the inverted pendulum with the proposed controllers (MRC, MRC+TIF, and MRC+T2F).

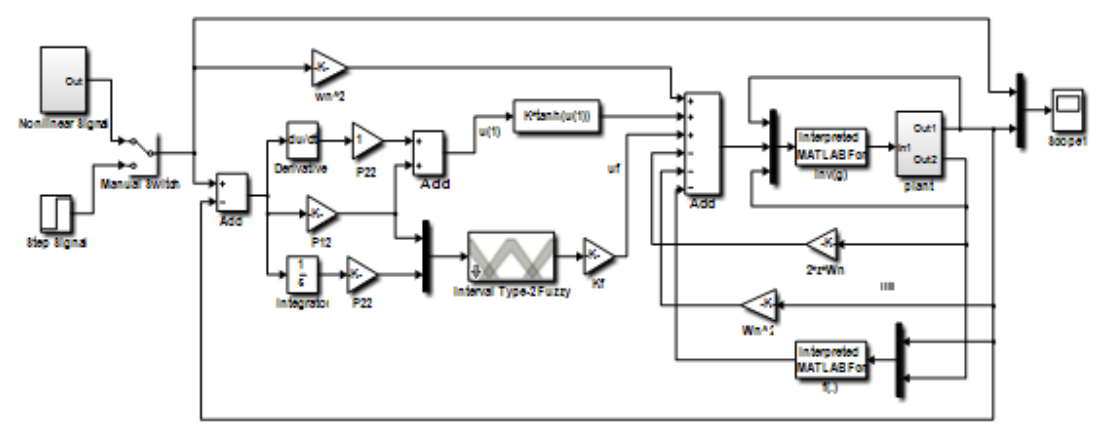

Figure 5. Complete matlab simulink connection for the nonlinear inverted pendulum with the suggested controller

The controller's parameters are given by Table 6 . Where designed paramters $K_{f}=2$ for linear trajectory and $K_{f}=20$ for nonlinear trajectory.

Table 6. The Controller's Parameters

\begin{tabular}{ccccccc}
\hline$P_{12}$ & $P_{22}$ & $K$ & $K_{f}$ & $\mathrm{~K}_{\mathrm{i}}$ & $\omega_{n}$ & $\zeta$ \\
\hline 14 & 2 & 100 & 2 or 20 & 10 & 10 & 0.5 \\
\hline
\end{tabular}


The different simulations are presented as follows:

\subsection{Simulation and analysis with linear trajectory (step signal) and uncertainty}

In the first simulation, a linear unit step input signal is used to test the overall inverted controlled system with the nonlinear disturbance of (25) and rules of Tables 3 and 5 for the fuzzy controller part. The results for this simulation with rule of Table 4 are shown in Figure 6, which show that the angular position tracking fairly good the desired input with no overshoot and nearly zero steady state error for the model reference controllers espatially with type-1 fuzzy scheme.

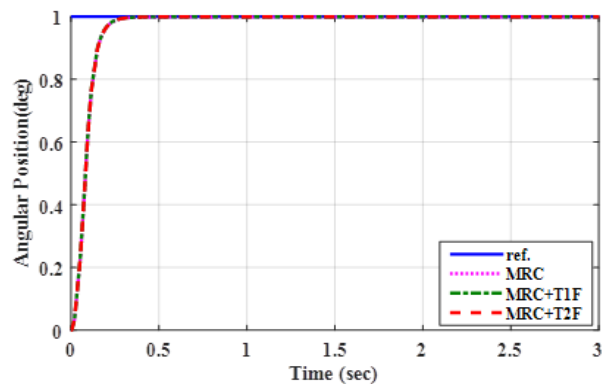

(a)

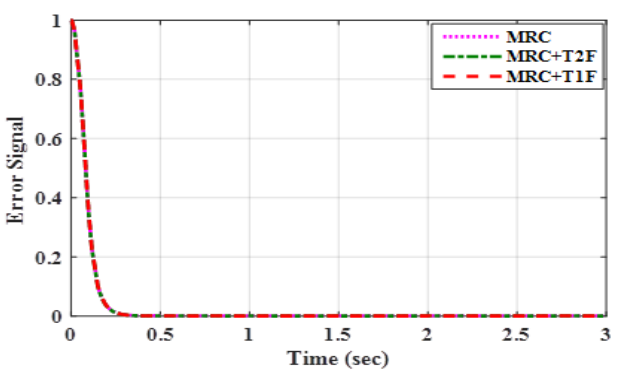

(c)

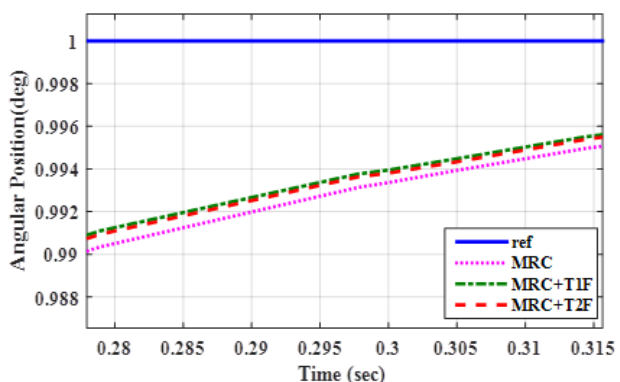

(b)

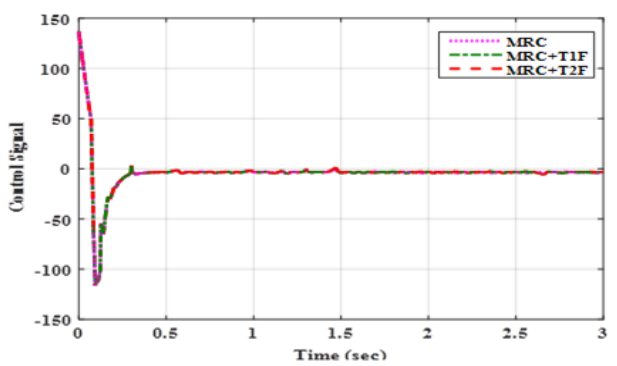

(d)

Figure 6. Simulation results for inverted pendulum controlled by (MRC, MRC+T1F, MRC+T2F) with step input (a) angler position; (b) zoom up the angular position; (c) error signal; (d) control signal

For this simulation, the performance of the inverted pendulum with (MRC, MRC+T1F, MRC+T2F) is evaluate by measure the indices steady state error $\left(e_{s . s}\right)$, settling time $\left(t_{s}\right)$, the scalar $M(t)$ at the steady state, in addition to integral of time multiplied by square error (ITSE) as illustrated by Table 7 which is show that the nonpositive condition of $M$ (Equation (22)) is satisfy for (MRC+T1F, MRC+T2F) and the proposed controller (MRC+T1F) is more efficient than (MRC) and (MRC+T2F) especially with rules of Table 4 because it makes the pendulum system flow the desired input signal very fast $\left(t_{s}\right.$ is small) and with a small ITSE as a comparison with (MRC) and (MRC+T2F).

Table 7. The pendulum performance comparison between the (MRC, MRC+T1F, and MRC+T2F)

\begin{tabular}{ccccc}
\hline Controller & $t_{s}$ & $e_{s . s}$ & $M$ & ITSE \\
\hline MRC & 0.2403 & 0.0002 & 0.0181 & 0.00248 \\
MRC+T1F with Table 5 & 0.2386 & 0.0008 & -0.0507 & 0.00247 \\
MRC+T2F with Table 5 & 0.2380 & 0.0009 & -0.0397 & 0.00249 \\
MRC+T1F with Table 4 & 0.2381 & 0.0003 & -0.0193 & 0.002463 \\
MRC+T2F with Table 4 & 0.2388 & 0.0004 & -0.0152 & 0.002478 \\
\hline
\end{tabular}

\subsection{Simulation and analysis with nonlinear trajectory (sinusoidal signal) and uncertainty}

In the second simulation see Figure 7 the nonlinear sinusoidal signal $r=\sin (0.5 \pi t)$ is used to test the controlled inverted system with nonlinear sinusoidal disturbance (25), results of this case are illustrate in Figure 7, these figures show that the inverted pendulum is remain stable with suitable control signal and very small error see in Figure 7(c) and 7(d) and the disturbance is remove from the response of the angular position with all controllers spatially (MRC+T2F). 


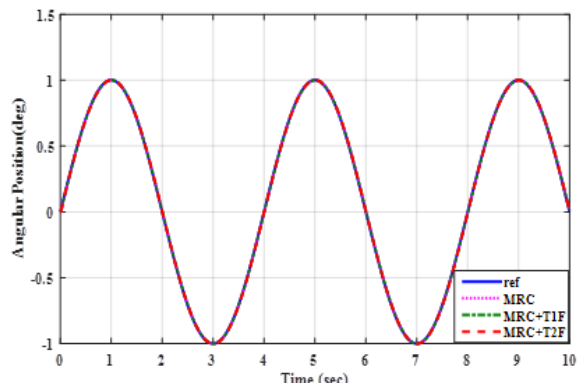

(a)

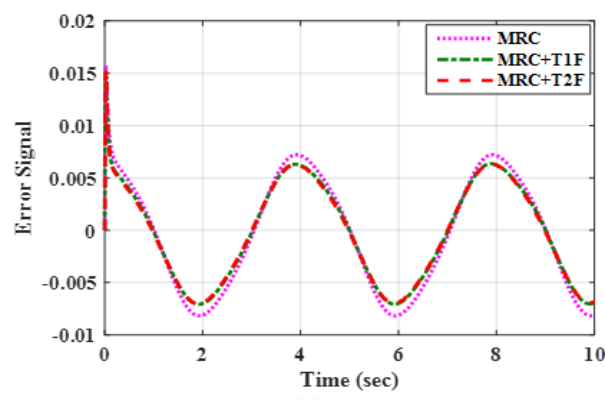

(c)

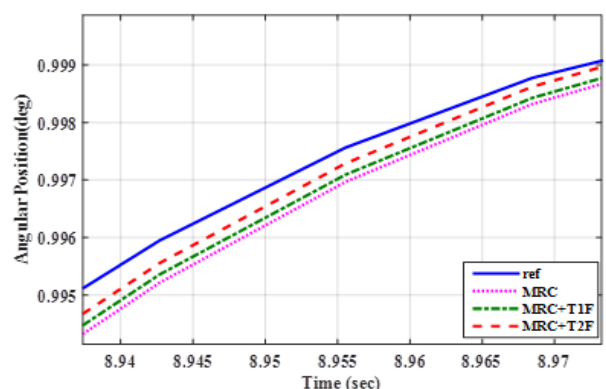

(b)

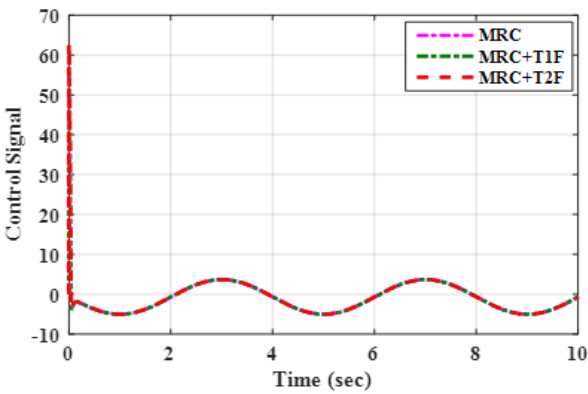

(d)

Figure 7. Simulation results for inverted pendulum controlled by (MRC, MRC+T1F, MRC+T2F) with nonlinear input signal, (a) angular position; (b) zoom up the angular position;

(c) error signal; (d) control signal

Table 8 show the $M(t)$ and ITSE after implement this simulation, it can be noted from this table that scalar $M$ is satisfy the negative condition with all controllers (MRC, MRC+T1F, MRC+T2F) and the $(\mathrm{MRC}+\mathrm{T} 2 \mathrm{~F})$ is more efficient than (MRC) and (MRC+T2F) especially with rules of Table 5 because it makes the pendulum system flow the desired input signal with small $M(t)$ and ITSE as a comparison with $(\mathrm{MRC})$ and $(\mathrm{MRC}+\mathrm{T} 1 \mathrm{~F})$.

Table 8. The Pendulum performance comparison with (MRC, MRC+T1F, and MRC+T2F)

\begin{tabular}{ccc}
\hline Controller & $M$ & ITSE \\
\hline MRC & -2.65 & 0.00144 \\
MRC+T1F with Table 5 & -1.9438 & 0.0008619 \\
MRC+T2F with Table 5 & -1.8786 & 0.0008128 \\
MRC+T1F with Table 4 & -2.1862 & 0.001092 \\
MRC+T2F with Table 4 & -2.1541 & 0.001063 \\
\hline
\end{tabular}

\subsection{Compare the results of the proposed controller with results of Ref. [44]}

The results of second simulation for the proposed controllers as shown in Figure 7 are compared with the results of the adaptive integral second order sliding mode controller [44] which are shown in Figure 8. It is clear from these results that, regardless of the performance of the required traceability, the control signal $u(t)$ suffers from unwanted chattering and its range traceability, the control signal $u(t)$ suffers from unwanted chattering and its range is bounded from (-20 to 20) while with proposed controllers, there is no chattering in control signal and the range of it is set between ( -5 to 5$)$ and this indicates the efficiency of the proposed controllers. 


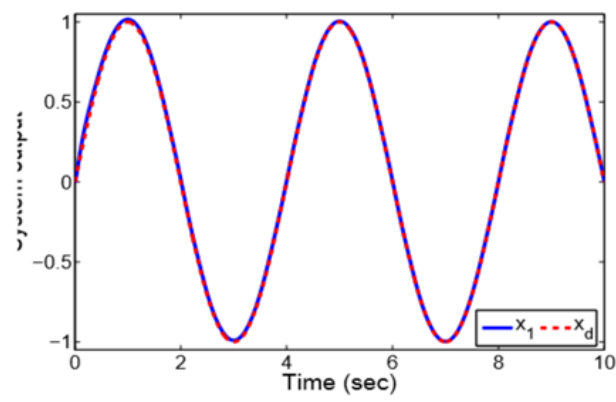

(a)

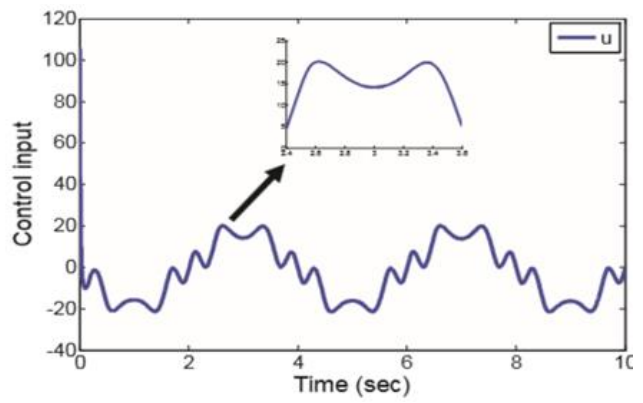

(b)

Figure 8. Simulation results for inverted pendulum with the proposed controller by [44] with nonlinear input signal, (a) angular position; (b) control signal

\section{SUMMARY AND CONCLUSIONS}

In this paper, an adaptive model reference controller with fuzzy type- 1 and 2 TSK is proposed for SISO nonlinear inverted pendulum system. The purpose of using fuzzy type-1 and 2 TSK is to improve the operation of the model reference controller and hence improve the performance of the inverted pendulum. By acquires the advantages of these two controllers, the steady state error is reduces and the uncertainty and disturbance is handled.

Experimental results demonstrate the efficiency of the proposed controllers (MRC+TIF, MRC+T2F) as a compare with model reference without fuzzy scheme and the adaptive integral sliding mode controller. Also, the results showed that the $(\mathrm{MRC}+\mathrm{T} 1 \mathrm{~F})$ is more efficient with the linear step input than the (MRC+T2F) with both case of the rules of Tables 3and 4. While for nonlinear input signal, the (MRC+T2F) is the most efficient with the both cases of the rules of Tables 3 and 4. As a future step we suggest applying the proposed controller to robotic systems such as manipulator, mobile robot, legged robot, or some robotic systems that can be used in different applications.

\section{REFERENCES}

[1] Souad and Benoit, "Model Reference Tracking Control for Nonlinear Systems Described by Takagi-Sugeno Structure," IET Control Theory and Applications, vol/issue: 1(5), pp. 1487-1495, 2007.

[2] Jiri and Petr, "Nonlinear Versus Ordinary Adaptive Control of Continuous Stirred-Tank Reactor," Scientific World Journal, 2015.

[3] H., "Nonlinear Systems," Prentice Hall, Upper Saddle River, New Jersey, second edition, 1996.

[4] S. and M., "Control Systems Design," $2^{\text {nd }}$ IFAC Conference, 2003.

[5] R. and A., "Nonlinear PI Control of Uncertain Systems: An Alternative to Parameters Adaptation," 5th IFAC, 2002.

[6] Byoung and Anthony, "Nonlinear Flight Control Using Neural Networks," Journal of guidance, control and dynamics, vol/issue: 20(1), 1997.

[7] Byung, et al., "Design and Stability Analysis of Single-Input Fuzzy Logic Controller," IEEE Transactions on Systems, Man, and Cybernetics Part B: Cybernetics, vol/issue: 30(2), pp. 303-309, 2000.

[8] Y. Chen, et al., "Comparative Study of PID Type-1 and Type-2 Fuzzy Control for Nonlinear Systems," IOP Conference Series Materials Science and Engineering, vol. 428, 2018.

[9] A. S. Samosir, et al., "Modeling and Simulation of Fuzzy Loogic based Maximum Power Point Tracking (MPPT) for PV Application," International Journal of Electrical and Computer Engineering, vol/issue: 8(3), pp.1315-1323, 2018.

[10] S. Rezanezhad, "Design of Fuzzy Optimized Controller for Satellite Attitude Control by Two State actuator to reduce Limit Cycle based on Takagi-Sugeno Method," International Journal of Electrical and Computer Engineering, vol/issue: 4(2), pp. 303-313, 2014.

[11] A. and M., "Hybrid Fuzzy-PID based Control of a Twin Rotor MIMO System," Annual Conference on IEEE, 2006.

[12] Bahloul, "Type-2 Fuzzy Control for Twin Rotor (TRAM)," Kasdi Merbah University-Ouargla Faculty of New Technology for Information and Communication Department of Electronics and Communications, 2015.

[13] J. Huang, et al., "Interval Type-2 Fuzzy Logic Modeling and Control Oof a Mobile Two-Wheeled Inverted Pendulum," IEEE Transactions on Fuzzy Systems, 2017.

[14] H. Ying, "Important and Challenging Issues for Interval Type-2 Fuzzy Control Research," Type-2 Fuzzy Logic and Systems book, 2018.

[15] A. M. El-Nagar, et al., "Intelligent Control for Nonlinear Inverted Pendulum Based on Interval Type-2 Fuzzy PD Controller," Alexandria Engineering Journal, vol/issue: 53(1), pp. 23-32, 2014.

[16] Hak and Emanuele, "Control Design for Interval Type-2 Fuzzy Systems under Imperfect Premise Matching," IEEE Transactions on Industrial Electronics, 2013. 
[17] Hodeiseh, "A Simplified Architecture of Type-2 TSK Fuzzy Logic Controller for Fuzzy Model of Double Inverted Pendulums," Theory and Applications of Mathematics \& Computer Science, 2012.

[18] S. and R., "Classical-Neural Synthesis of Nonlinear Control Systems," Journal of guidance, control and Dynamics, vol/issue: 25(3), 2002.

[19] C. X. Jie, "Model-Reference Adaptive Control Based on Neuro fuzzy Networks," IEEE transactions on systems, man, and cybernetics-part c: applications and reviews, vol/issue: 34(3), 2004.

[20] Zirkohi and M. A. Moradi, "Model reference type-2 fuzzy sliding mode control for a novel uncertain hyperchaotic system," Journal of Intelligent 7\& Fuzzy Systems, vol/issue: 32(1), pp. 389-400, 2017.

[21] J. J. Slotine and W. P. Li., "Applied Nonlinear Control, Engle- wood Cliffs," NJ, Prentice Hall, 1991.

[22] F. Qiao, et al., "Adaptive Sliding Mode Control based on Fuzzy Logic Scheme," Faculty of Computing, Engineering and Mathematical Sciences, 2004.

[23] Katsuhico, "Modern Control System," University of Minessota, printed in the United States of America, pp. 1-1013.

[24] J. M. Mendel, "Uncertain Rule-Based Fuzzy Logic System: Introduction and New Directions," Prentice Hall, Upper Saddle River, NJ, USA, 2001.

[25] J. M. Mendeland and R. I. B. John, "Type-2 Fuzzy Sets Made Simple," IEEE Transactions on fuzzy systems, vol/issue: 10(2), pp. 117-127, 2002.

[26] N. and J., "Applications of Type-2 Fuzzy Logic Systems to Forecasting of Time-Series," Information sciences, vol/issue: $120(1)$, pp. 89-111, 1999.

[27] Niranjan, et al., "Non-Linear Control and Stabilisation of VSC-HVDC Transmission System Based on Type-2 Fuzzy Sliding Mode Control," International Journal Automation and Control, vol. 7, 2013.

[28] Hossein and Jafar, "Interval Type-2 Fuzzy Logic Controller Design for the Speed Control of DC Motors," Systems Access Journal, vol. 3, pp. 266-273, 2015.

[29] Rahib, "Credit Rating Using Type-2 Fuzzy Neural Networks," Hindawi Publishing Corporation Mathematical Problems in Engineering, 2014.

[30] Ahmet and Tufan, "An Open Source Matlab/Simulink Toolbox for Interval Type-2 Fuzzy Logic Systems," Conference of IEEE Symposium Series on Computational Intelligence, Turkey, 2016.

[31] Rim, et al., "Adaptive Type-2 Fuzzy Second Order Sliding Mode Control for Nonlinear Uncertain Chaotic System," International Journal of Computational Science, Information Technology and Control Engineering (IJCSITCE), vol/issue: 2(4), 2015.

[32] T. and M., "Fuzzy Identification of Systems and Its Applications to Modeling and Control," IEEE Transactions on Systems, Man, and Cybernetics, vol/issue: 15(1), pp. 116-132, 1985.

[33] Nesrine, et al., "Interval Type-2 Fuzzy Logic Control of Mobile Robots," Journal of Intelligent Learning Systems and Applications, pp. 291-302, 2012.

[34] Q. and J., "Equalization of Nonlinear Time-Varying Channels Using Type-2 Fuzzy Adaptive Filters," IEEE Transactions on Fuzzy Systems, vol/issue: 8(5), pp. 551-563, 2000.

[35] Allaoua and Laoufi, "Novel Sliding Mode Fuzzy Control Based on SVM for Electric Vehicles Propulsion System," ECTI Transactions on Electrical Engineering, Electronics and Communications, vol/issue: 10(2), 2012.

[36] Xiaoyu, "Sliding Mode-Like Fuzzy Logic Control with Adaptive Boundary Layer for Multiple-Variable Discrete Nonlinear Systems," Journalof Intelligent Systems, pp. 209-220, 2016.

[37] Bahlool, et al., "Type-2 Fuzzy Control for Twin Rotor (TRMS)," Kasdi Merbah University-Ouargla Faculty of New Technology for Information and Communication Department of Electronics and Communications, 2015.

[38] http://exploration.grc.nasa.gov/education/rocket/rktstab.html

[39] C. Wang, et al., "Direct Adaptive Fuzzy-Neural Control with State Observer and Supervisory Controller for Unknown Nonlinear Dynamical Systems," IEEE Transactions on Fuzzy Systems, vol/issue: 10(1), 2002.

[40] H., "Nonlinear Systems," Third Edition, Prentice Hall, 2002.

[41] JinKun L. and Fuchun S., "Chattering Free Adaptive Fuzzy Terminal Sliding Mode Control for Second Order Nonlinear System," Journal of Control Theory and Applications, pp. 385-391, 2006.

[42] W. D. Chang and S. P. Shih, "PID Controller Design of Nonlinear Systems Using An improved Particle Swarm Optimization Approach," Communications in Nonlinear Science and Numerical Simulation, vol. 15, pp. 3632-3639, 2010.

[43] Wu, "Adaptive Fuzzy Sliding Mode Control for Inverted Pendulum," Proceedings of the Second Symposium International Computer Science and Computational Technology (ISCSCT'09) Huangshan, pp. 231-234, 2009.

[44] Sanjoy, "Adaptive Second Order Sliding Mode Control Strategies for Uncertain Systems," PhD thesis, Indian Institute of Technology Guwahati, Department of Electronics and Electrical Engineering, India, 2012.

[45] Leila, et al., "Sliding Mode Trained Neural Control for Single and Coupled Inverted Pendulum System," Researches and Applications in Mechanical Engineering, vol/issue: 2(4), 2013. 